\title{
Emotion and Consumption Profiles in a COVID-19 Environment
}

\author{
Melissa Han \\ 3115 Windsong Drive, Oakton, VA, 2212,USA; melissahxy02@gmail.com
}

ABSTRACT: Since the outbreak of COVID-19, the world has learned how to live during and respond to a pandemic. The emotional trauma and confusion people are experiencing lead to behavioral changes during this unprecedented time. This paper aims to study people's emotional and purchasing pattern changes after the COVID-19 outbreak. The survey results revealed that $83.9 \%$ of all respondents decreased their shopping at grocery store while $60.5 \%$ increased online shopping. Additionally, significant increases occurred in negative emotions such as being frightened, annoyed, and anxious. This increase, in turn, led to the changes in purchasing amounts of toilet paper, bags of rice, and face masks. The data reflected that the respondents' purchasing patterns are necessity driven.

KEYWORDS: Biology; Coronavirus; COVID-19; Emotions; Consumption patterns; China; Survey; Health; Economics.

\section{- Introduction}

The 2019-identified Coronavirus COVID-19 has changed the way the world acts as infection rates and death tolls continue to rise even under recommended and mandatory regulations. As of September 7th, 2020, there have been 27,249,308 confirmed cases and 890,971 confirmed deaths worldwide. ${ }^{1}$ Over the week beginning August 31st to September 7th, 2020, there has been an average of 40,525 reported cases per day in the United States. ${ }^{2}$ As of September 7th, 2020, the U.S. has $6,300,075$ reported cases and 189,182 confirmed deaths since the virus was first identified on January 21st, 2020. ${ }^{3}$

The overwhelming spread of the virus and the multiple impacts of coronavirus increase anxiety and uncertainty. Mandatory health orders, while decreasing the possibilities of infection, increase isolation, stress, and fear. ${ }^{4}$ Additionally, since the COVID-19 virus outbreak, countries have experienced unexpected unemployment levels, which heavily affected their economies. The International Labour Organization (ILO) reports "over one in six young people surveyed have stopped working since the onset of the COVID-19 crisis." The ILO also states that working hours have declined by " 10.7 percent relative to the last quarter of 2019 , which is equivalent to 305 million full-time jobs." ${ }^{5}$ Since mid-March, the United States has lost 20.6 million jobs, corresponding to an unemployment rate of $14.7 \%$, a level not seen since the Great Depression in the 1930s. ${ }^{6}$ This significant drop in employment rates and the huge hit on economies have significantly raised stress and anxiety levels.

The new normal of living during this pandemic - quaran ${ }^{-}$ tine and social distancing, alertness of contact and possible infection, reduction in leisure activities, financial and economic instability - increases stress levels. ${ }^{7}$ This escalated uncertainty and fear has led to changes in the purchasing profiles of people. For instance, certain people purchase more than they need in response to fear, and certain people restrict the consumption and purchases of goods in fear of, one day, not having enough monetary savings.
In this paper, Section II reviews various research and reports discussing and analyzing topics regarding the impact of infectious diseases and public health crisis on emotions and purchasing behaviors. Section III discusses the results gathered from a survey. The outcomes are grouped into multiple trends; some are aligned with our assumptions while some are not. Section IV analyzes the underlying reasons for why the results are the way they are. Additionally, it examines the connection between the results and economics. Section $V$ reflects on the accomplishments and limitations of the project and survey. Lastly, Section VI concludes the paper by connecting the findings of this paper to a broader context and future references.

This paper studies the change and relationship between emotions and purchasing patterns during the uncertain time caused by COVID-19. How has COVID-19 influenced emotional patterns? How have those emotional changes affected purchasing behaviors? What type of emotion most heavily influences purchasing patterns during the uncertain times caused by COVID-19?

\section{Literature Review:}

Previous papers and articles have researched the impact of infectious diseases on human emotions; other papers have investigated the influence of emotions on purchasing patterns. These previous papers form the theoretical foundation of this paper.

Restubog, Ocampo, and Wang ${ }^{8}$ (2020) showed the consistent relationships between an outbreak of infectious disease and chain-reactions of psychological and behavioral consequences. Within their commentary, they highlight the negative psychological effects such as greater incidents of depression and psychological distress, worry, anxiety about being infected, and subjective well-beings.

Additionally, McGinty, Presskreischer, Han, and L. Barry9 (2020) compared their April 2020 survey results regarding symptoms of psychological distress and loneliness within U.S. adults with national data from 2018. Their resulted comparison -13.6\% of April 2020 surveyed U.S. adults reported signs of 
serious psychological distress compared to the $3.9 \%$ in 2018 - further exemplifies the psychological and emotional strain COVID-19 has caused.

McGinty, Presskreischer, Han, and L. Barry (2020) recount that along with other stressors such as fear of contracting the disease and uncertainty for the future, the necessary mitigation strategies such as those imposed for COVID-19 (e.g., social distancing, home quarantine, and travel restrictions) can negatively affect mental health.

Jin, Song, Zhao, and $\mathrm{Yao}^{10}$ (2020) investigated the impact of COVID-19 on the purchasing behavior of Chinese citizens. They unveiled that by increasing the risk perception, fear, and boredom of citizens, COVID-19 accelerates purchases based on bandwagon effects, impulsive purchasing, and scarcity level.

One especially interesting report from Lee and Ward ${ }^{11}$ (2020) demonstrates the increase of substance use in response to increasing distress among people. Lee and Ward (2020) report on the shift in the social relationships and increase in distress of people during the Coronavirus pandemic. To demonstrate, Lee and Ward surveyed 562 adults and found that $50 \%$ or more reported symptoms of anxiety nearly every day or several days a week since COVID-19.28\% of all respondents' report uses of alcohol or other drugs to make themselves feel better.

Based on these reports, this paper explores further the relationship between COVID-19 and emotions and purchasing patterns. Additionally, the paper analyzes the surveyed results with various demographic factors such as age and living country to explore alternative influences that might impact the emotions and behaviors of people.

\section{Results}

A total of 187 respondents completed the surveys, with 47 respondents from the English version and 140 from the Chinese version. The respondents range from ages of 12 to 64 . The survey participants comprise of $40.7 \%$ male respondents and $59.3 \%$ female respondents. Additionally, the respondents indicated their educational level and their annual household income. These demographic-targeting questions aim to demonstrate the background information of respondents. Additionally, these questions were used to discover correlations between factors such as age and COVID-19-related responses from the surveyed groups.

The results collected display multiple noticeable changes in the psychological and behavioral responses of the surveyed groups. Most of these changes align with the predictions of the research, while others stray away due to both expected and unexpected factors.

The first pattern is the increase in intensity of negative emotions since the outbreak of COVID-19. Respondents were asked to rate the intensity of 8 positive and negative emotions on a scale of 0 (least intense) to 10 (most intense). The responses demonstrate that negative emotions such as being frightened, annoyed, and anxious display an increase in intensity from before the outbreak. Positive emotions such as excited, calm, and confident present a decrease in intensity. Figure 1 documents the difference between rated intensities of emotions from the respondents. The most noticeable change between ratings is the emotion "frightened." Of the 187 respondents, the average rating of the intensity of the emotion "frightened" was 2.6 before the outbreak of COVID-19. When asked to rate the same emotion, based on emotional experiences post COVID-19 outbreak, this average rating increased to 4.4 .

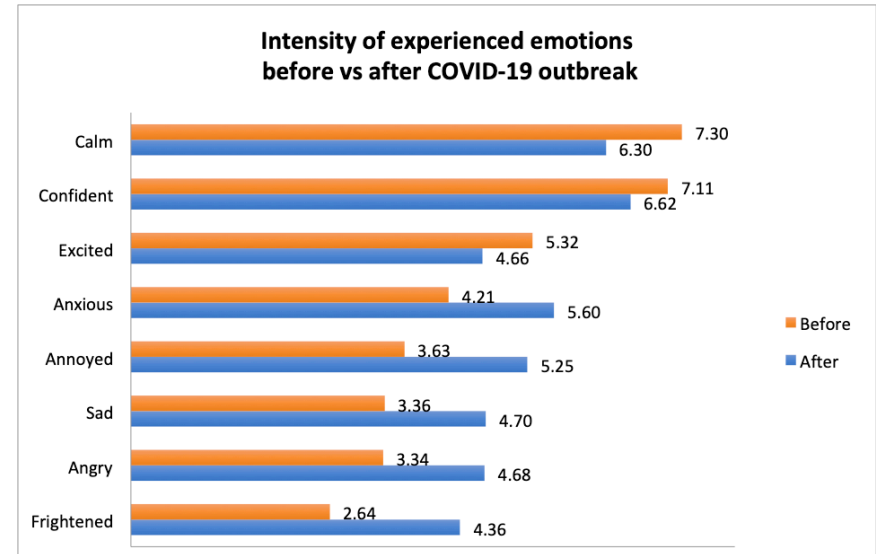

Figure 1: Intensity of experienced emotions before vs after COVID-19 outbreak. The intensity of all three positive emotions decreased while the five negative emotions increased. The three emotions with largest difference from before to after COVID-19 outbreak were Frightened, Annoyed, and Anxious.

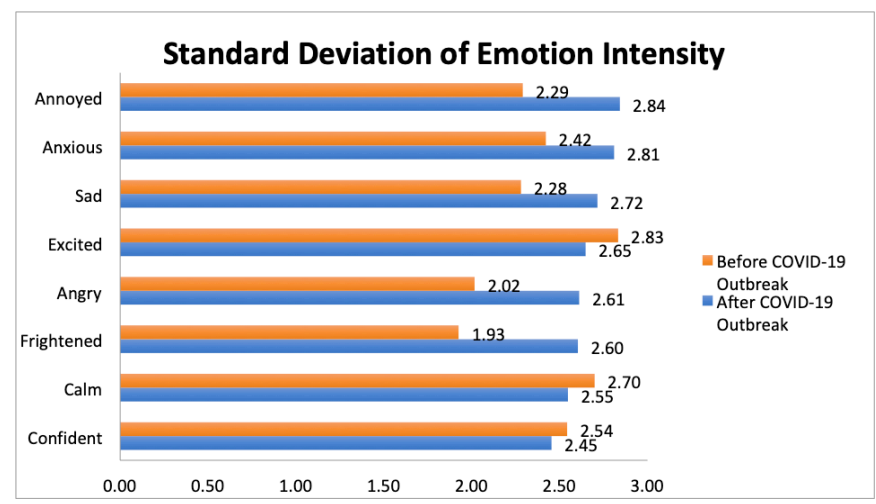

Figure 2: Standard Deviation of Intensity of experienced emotions before vs after COVID-19 outbreak. Demonstrates the standard deviation of the intensity of each emotion.

The second pattern, displayed through Figures 3, 4, and 5, demonstrated that people have decreased their overall consumption of goods and shopping at grocery stores while increasing their online grocery shopping: $44.6 \%$ of all respondents decreased their overall consumption of goods; $83.9 \%$ of all respondents shop at grocery stores less frequently; $60.5 \%$ of all respondents increasing their online grocery shopping.

The third pattern is demonstrated through comparing the shopping influences from before and after the outbreak of COVID-19. The survey asks the respondents to choose the three most important factors they base their purchases on when shopping. As expected, displayed by Figure 6, the choices such as level of necessity, affordability, and availability increased in chosen frequency for the post-COVID-19 outbreak. In contrast, choices such as personal satisfaction, brand reputation, and design uniqueness decreased in frequency. Due to the outbreak of COVID-19, the determining factors change as priorities shift from personal preferences to level of necessities and safety oriented. 
Overall consumption of goods after COVID-19 outbreak

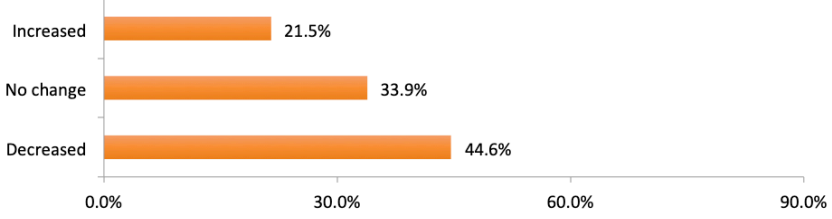

Figure 3: Change in overall consumption of goods after COVID-19 outbreak. Almost half of the respondents (44.6\%) decreased overall consumption of goods, while $21.5 \%$ increased overall consumption of goods.

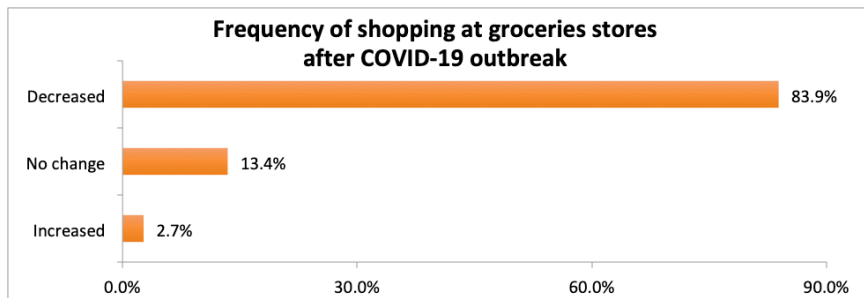

Figure 4: Frequency of shopping at groceries stores after COVID-19 outbreak. Most of the respondents (83.9\%) decreased grocery store shopping.

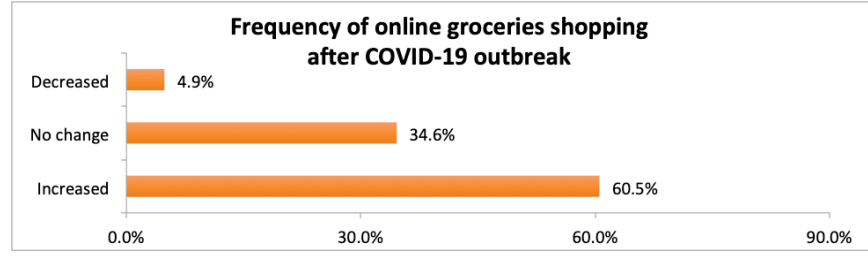

Figure 5: Frequency of online groceries shopping after COVID-19 outbreak. Over $60 \%$ of the respondents increased online grocery shopping.

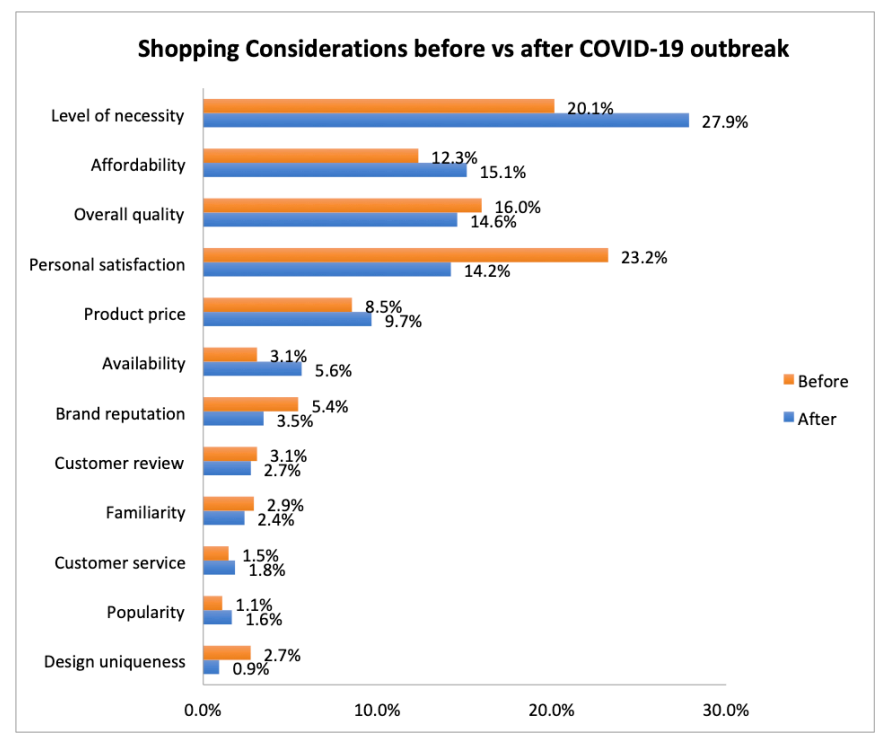

Figure 6: Shopping considerations before vs after COVID-19 outbreak. The influences of Level of Necessity, Affordability, and Availability increased for post-COVID-19 outbreak, while Personal Satisfaction, Brand Reputation, and Design Uniqueness decreased.

The fourth pattern is identified through the questions targeting changes in purchased amount of toilet paper (English version only) and bags of rice (Chinese version only). When designing the English version survey, it seemed necessary to include questions that could reflect whether the respondents increased their toilet paper stock due to COVID-19. It was publicized that in response to the outbreak of COVID-19, many Americans stocked up on- in addition to other essentialsrolls of toilet paper. Media outlets such as TIME ${ }^{12}$, Nature ${ }^{13}$, and $\mathrm{NBC}^{14}$ news have reported on the massive amount of toilet paper buying. To test this behavior, the surveys provided the questions "approximately, how many rolls of toilet paper do you currently have in stock for yourself?" and "did you increase your stock of toilet paper due to COVID-19?"'The results align with the toilet media-reported paper-buying phenomenon: Figure 7 demonstrates that $54.3 \%$ of all United States respondents stated they increased their stock by a certain percentage while $45.7 \%$ of all respondents stated they did not increase their stock of toilet paper.

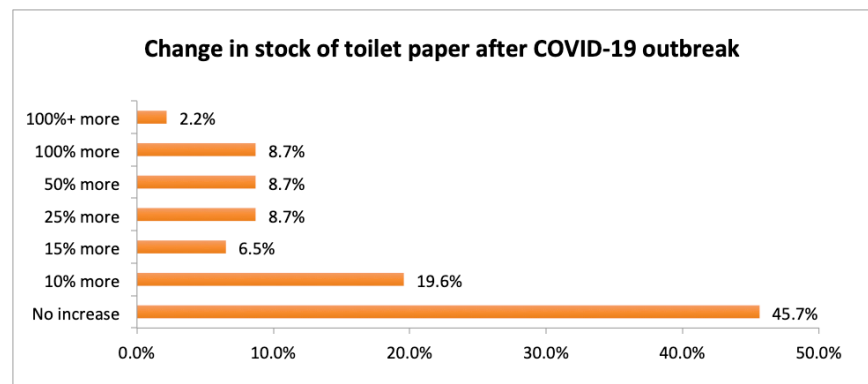

Figure 7: Change in stock of toilet paper after COVID-19 outbreak. 54.3\% of the United States respondents increased their toilet paper stock while $45.6 \%$ did not.

While many Americans stocked up on toilet paper, the Chinese version of the survey targeted what was thought to be an essential to save for Chinese people - rice. Surprisingly, the results display that $65.0 \%$ of the respondents who answered the Chinese version did not increase their stock of bags of rice. Figure 8 displays the data from further analysis of this surprising phenomenon. To investigate, the results from the question "did you increase your stock of rice due to COVID-19" were analyzed based on the reported living location. It is hypothesized that maybe their current living location might impact why they did not stock up on rice. The eating habits of Chinese culture show that people who live in southern China consume more rice while those living in northern China consume more flour. Therefore, it was hypothesized that the majority of the respondents came from northern China due to result in the lack of savings in stock of rice. After further study, the data demonstrate that, despite the larger number of respondents not increasing their rice stock, there was still 8.7\% more southern China respondents who reported increasing rice stock than northern China respondents (shown in Figure 7). This difference still confirms the previous assumption based on Chinese cultural eating habits but implies that there are other reasons leading to the reported outcome documented in Figure 8.

Finally, the fifth pattern is the differences and similarities between the number of masks people in China or America bought. The survey asks all participants to approximate the number of masks they have both for themselves since the outbreak of COVID-19. After summarizing the responses, a few patterns can be seen (through Figure 9). 


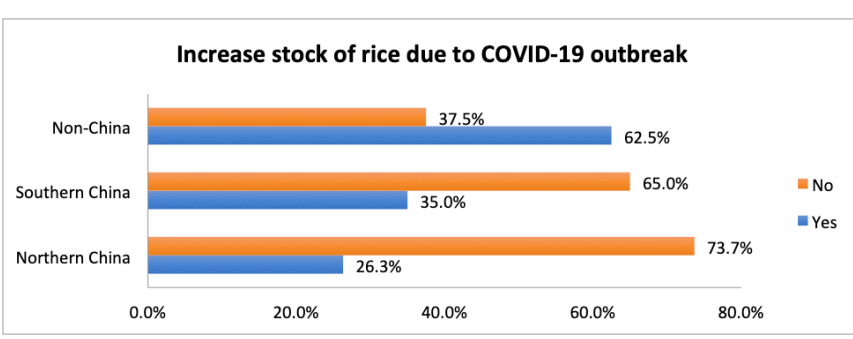

Figure 8: Increase stock of rice due to COVID-19 outbreak. $62.5 \%$ of respondents living outside China increased their stock of rice. Compared to respondents living in Northern China, an increase of $8.7 \%$ Southern Chinese respondents increased their stock of rice.

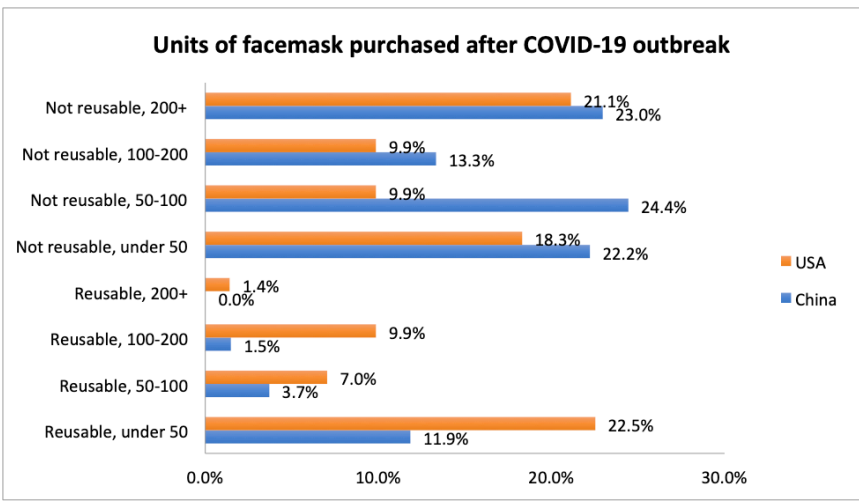

Figure 9: Units of facemask purchased after COVID-19 outbreak.

Firstly, the data suggests that people who live in China prefer purchasing non-reusable masks more than reusable masks. When given the same masks unit, more respondents stated they bought them in a non-reusable form than reusable. Secondly, respondents who live in the United States seem to purchase more reusable masks. More percentage of United States participants purchased a certain unit of reusable masks than did participants who live in China. For instance, 9.9\% of United States respondents purchased 100-200 units of reusable masks when only $1.5 \%$ of Chinese respondents purchased the same type and amount. Similarly, respondents who live in China purchase more non-reusable masks of the same unit than did United States respondents.

\section{- Discussion \\ 1.Why did the most intense emotions change the way \\ they did? :}

As shown in Figure 1, the comparison between rated intensities of emotions from before COVID-19 to after COVID-19 demonstrates an increase in negative emotions and decrease positive emotions. It is expected that people have experienced various negative emotions more frequently and intensely. According to the survey, many respondents accounted for their increased intensity of anxious, sad, frightened, annoyed, and angry on the fears of infection, societal disorder, and national economic downturn (shown in Figure 9). The increase in such negative emotions, in turn, leads to many changes in purchasing patterns.

\section{What accounts for the overall change in grocery purchasing behavior?:}

Based on the second identified pattern and Figures 3 through 5 , there is a decrease in overall consumption of goods and shopping at supermarkets while online grocery shopping increases. People visit grocery stores less and opt for online shopping as much as they can to avoid possible contact with other people. The fear of going outside and being infected leads to lesser shopping at supermarkets. The respondents imply that, due to COVID-19, they only go grocery shopping if it is necessary. To compensate for lesser visits at the grocery stores, people would turn to online shopping. Although there were questions of whether one can be infected if receiving a package, the respondents seem less frightened to shop online compared to shopping in person.

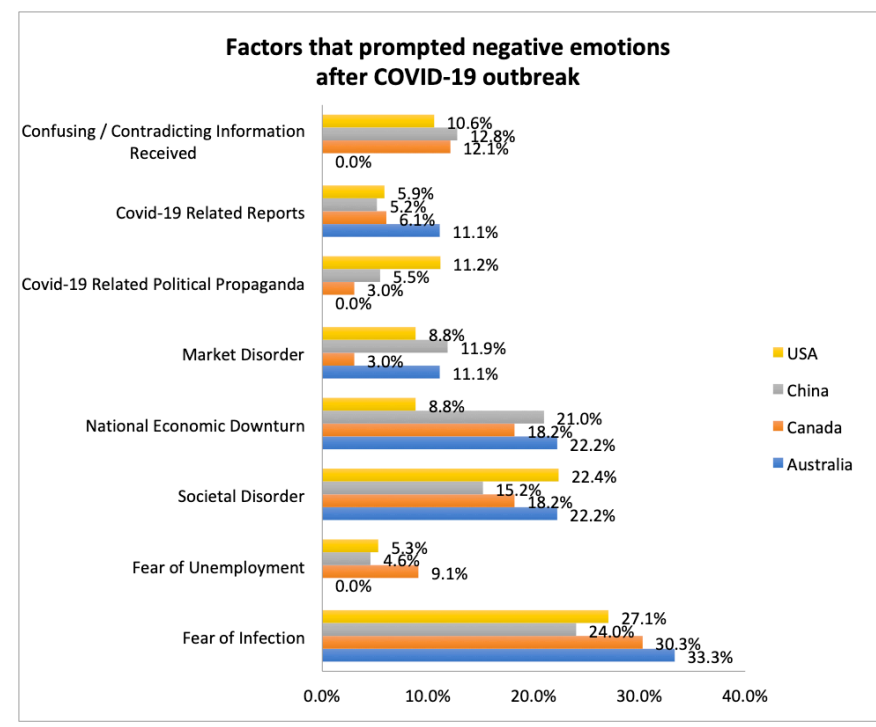

Figure 10: Factors that prompt negative emotion after COVID-19 outbreak. Fear of Infection and Societal Disorder are the top 2 factors prompting negative emotions regardless of living countries.

\section{Why did more people choose "the level of necessity,"} "affordability," and "overall quality" as the three most important factors when shopping post COVID-19 outbreak? : The outbreak of COVID-19 changed the demands of people. Before the pandemic, emotional needs and wants likely influenced people's purchasing intention. However, as the pandemic intensified, the needs of people shifted to necessity driven and realistic purchases.

When asked to choose the three most impactful factors when shopping after the outbreak of COVID-19, the choices that had the highest amount of increased chosen percentage, as shown in Figure 6, were "the level of necessity," "affordability," and "availability." In contrast, the choices that had the highest amount of decreased chosen percentage were "personal satisfaction," "brand reputation," and "design uniqueness." This result is quite expected - amid a global pandemic, the purpose and determining reasons of a purchase is more constricted and concise. Due to the pandemic, people make more rational choices since they are more concerned on price factors than before the outbreak. Almost all purchases are made with the effects of the pandemic in mind.

Firstly, many of the purchases made after the outbreak were for combating the disease. People are afraid of getting infected, so they purchase what is necessary for protection. This fear leads to the increase in the level of necessity, which is reflect 
ed in the response. Additionally, with one of the main fears being national economic downturn, many might save their money anticipating instable income flow and large amounts of layoffs and business closings since the outbreak. With more constraints on their money, the demand for luxuries decreases while the demand for necessities increases. Luxuries are never necessary: they are items that help improve the quality of living but are not indispensable for living. As people begin to live amid a pandemic, the need for luxuries decrease and the level of necessities increase.

"Affordability" works in the same fashion as "level of necessity." During COVID-19, many of the purchases are carefully thought out. People must take into consideration whether they can afford a purchase during times with inconsistent income flow. The fear of not having enough savings prompts people to reconsider when they face a purchase that affects their financial stability. The income level and affordability of a person is, therefore, another determining factor of whether they make a purchase or not.

Once people have concluded that they are able to afford a purchase, the issue of "availability" then arises. Many items previously not considered essential, are now prioritized. For instance, many respondents have reported an increase in their stocks of toilet paper and purchases of large amounts of masks. Due to this sudden increase in demand, the supply cannot catch up to the demand. Therefore, there are frequent shortages in these items. With this type of situation frequently occurring, people proceed to purchase based on availability. As soon as they see these necessities are available, people might purchase large amounts of it in fear of having to buy more when they have shortages.

With fear and anxiety influencing people during the purchasing process, factors such as "personal satisfaction," "brand reputation," and "design uniqueness" are not as influential as before. In the face of a pandemic, previously enjoyed purchases that satisfy these three factors are probably eliminated as people are focusing on protection, safety, and health.

\section{Why did the United States respondents stock up on toilet paper? :}

First, it is necessary to understand why toilet paper is a main item of saving up since the outbreak of COVID-19. Toilet paper is a necessity that every person uses daily. What makes it even more important is that there are no close substitutes for toilet paper and humans are long past the years where primitive cleaning methods are still in use. Therefore, with no substitutes for toilet paper, people, reasonably, increased their stock of toilet paper when informed they will be quarantined. Besides this reason, other psychological factors also prompted the massive amounts of saving on toilet paper.

The first psychological impact, according to TIME, is that it is not the toilet paper itself that draws customers; it is the idea that there are inadequate supplies that frightens and, therefore, attracts people. With toilet paper already being a necessity that people need to purchase, the fact that there is a lack of it increases the anxiety within people. According to the idea of scarcity, the scarcer something is, the more valuable and attrac According to the idea of scarcity, the scarcer something is, the more valuable and attractive it is. ${ }^{15}$ Knowing that there is a lack of toilet paper scares people, especially since toilet paper is something irreplaceable.

The second psychological impact comes from the fear of missing out, or FOMO, the modern expression for herd psychology. According to Michigan Live ${ }^{16}$ and $\mathrm{BBC}^{17}$, the fear of missing out is the reason why toilet paper is flying off the shelves at grocery stores. When people start "panic-buying," others tend to follow because they fear they will not get any for themselves. Additionally, people might think, seeing the amounts of reports of people stacking up toilet paper, that there is a reason for people to purchase toilet paper, leading them to believe they must have it. This phenomenon is especially magnified when the item people are panic buying is considered necessary and irreplaceable. ${ }^{16}$

Both influences are driven by fear of not having enough of something irreplaceable, and the wide media coverage on the lack of toilet paper does not help control it. With publication about toilet paper, and regardless of whether one fears missing out or falls into the notion of scarcity, the result is the same: more and more people purchase stacks of toilet paper, leading to its lack of supply.

\section{Why did respondents who live in China not save up on rice in the same way as United States respondents for toilet paper? :}

When it comes to the amount of rice savings, there is a fundamental difference between rice and toilet paper: the two items the survey framed as necessities. Both these items are necessities because people must use toilet paper daily, and many people living in China consume carbohydrates like rice daily. However, the fundamental difference between the two is that toilet paper does not have substitutes, but rice does. People can consume different types of carbohydrates in replacement of rice if they do not have any. Unfortunately, this difference was not considered when creating the survey. It was presumed that rice would act in the same way as toilet paper does, when in fact it had substitutes, leading to a lesser level of necessity. Therefore, this difference is the reason for the results demonstrated in Figure 8.

The fact that fewer people are stocking up on rice in China than there are outside of China reflects a few reasons. Firstly, at the time of the survey (July $12^{\text {th }}, 2020$ to July $26^{\text {th }}, 2020$ ), the infection rates of COVID-19 in China have already been controlled. Even after the second wave of outbreak in Beijing that began in June 2020, infections were quickly contained. After July 5th, no new cases were detected. ${ }^{18}$ With effective containment and lower cases, the fear and the need to stock up on essentials might have decreased, leading to the results summarized in Figure 8. Lower infection rates equate to fewer negative emotions such as fright and fear that could prompt changes in purchasing patterns.

Secondly, when looking at Americans purchasing toilet paper, media outlets have greatly impacted the actions of people saving up toilet paper. With more media reports on toilet paper running out of stock more people go buying them. With this cycle snowballing bigger and bigger, the demand for toilet paper increases while the supply is still trying to catch up. ${ }^{19}$ 
Comparatively, the media within China is controlled, especially during a pandemic, in order to minimize any possible fear and fright within the society. Any story or information that might increase anxiety and fear is controlled in order to main societal order. Therefore, there were little to none reports on whether people are purchasing more bags of rice or not. With no media reports of rice running out of stock, people might not feel the fear of possibility lacking a necessity.

\section{Why do United States respondents tend to purchase reusable masks while respondents who live in China buy non-reusable ones? :}

Figure 8 shows that United States respondents tend to purchase more reusable masks than the respondents who live in China. This occurrence can be explained through a few possible reasons. Firstly, it might be presumed that, compared to reusable masks, non-reusable masks and medical masks are better at combating against COVID-19. Throughout the pandemic, there have been specialists who stated that disposable masks are effective in combating the virus so long as they are worn properly. ${ }^{20}$ Additionally, although many have chosen to use reusable masks, to many people who live in China where populations are crowded and cities are much more compact, a reusable mask might have more risks than a non-reusable mask Since people dispose of non-reusable masks as soon as they finish using it, people might believe that is safer. In China, it is possible that citizens are much more anxious and concerned as COVID-19 was first discovered there. With months of combating the disease on their own, it is possible that the citizens are much more cautious in protecting themselves against infection. In result, this fear and anxiety of possible contamination might lead to more purchases of non-reusable masks.

On the other hand, COVID-19, although having the highest reported death toll in the United States at this moment, seems to stir less fear in America. According to a July Axios-Ipsos poll, almost one in three Americans believes that the death toll of the COVID-19 is not as high as the official counts. ${ }^{21}$ Also, according to a March NPR PBS NewsHour Marist poll, about $56 \%$ of Americans think COVID-19 is a real concern and threat, a 10\% drop since February. The poll also states that a growing number of Americans think the coronavirus is being "blown out of proportions. ${ }^{22}$ With growing suspicion and disbelief, it is possible that some of the surveyed United States participants simply thought there is no need to purchase much of non-reusable masks because they, in turn, are not that anxious and frightened of the new pandemic.

\section{Reflection:}

Overall, the survey helped provide a great basis of data that allowed for extensive analysis. The discovered relationship gave insight into the emotional and purchasing changes that occurred so far during an infectious pandemic. However, there were a few issues that could improve the analysis and conclusions if resolved. Firstly, when finding participants for the survey, the groups from each country were not controlled, leading the number of people from each country to be different. Although this still provided sufficient data, this difference could affect the conclusions if the numbers of each groups were controlled. For instance, the data currently shows that people who live in America tend to purchase reusable masks due to, possibly, environmental consciousness, affordability, and availability. However, since there were only 47 United States respondents, this conclusion could change if more participated. Additionally, there were only three participants from Australia and 11 participants from Canada. With such small numbers of participants from these two countries, it was not accurate and correct to analyze data with such a small pool of respondents. Similarly, because the surveyed group was not controlled and all of them were volunteers, the surveyed groups demonstrated an overall higher household annual income level (shown in Figure 10). It was expected that one of the highest chosen negative-emotion-prompting factors was fear of employment. However, since the surveyed group had a higher income level, this assumption was not demonstrated. Once again, this shows that the uncontrolled survey group has brought an amount of

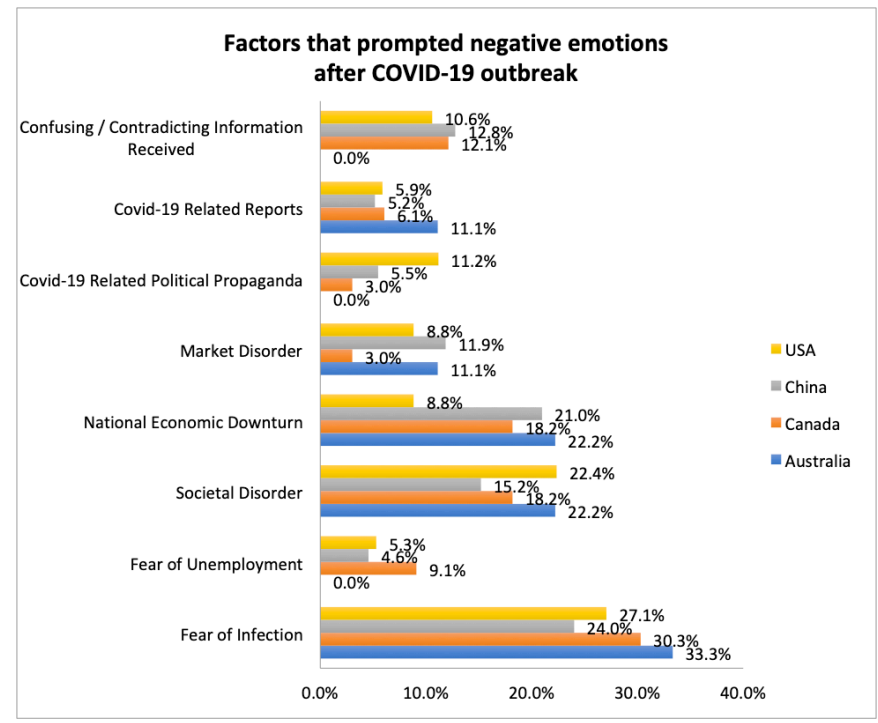

Figure 11: Respondent household annual income. $75.1 \%$ respondents' annual income is above $\$ 50 \mathrm{~K}$.

bias into the conclusion.

Another error-prompting decision was comparing toilet paper to rice as the essential good that people purchase in China. Toilet paper is irreplaceable, but rice is not. Therefore, rice might not lead to the best comparative results even though it generated a great amount of analysis.

Additionally, according to feedback, some questions had confusing wording, which could have led to inaccurate and invalid data. For instance, the question asking the participants to rate the experienced intensity of 8 emotions out of 0 to 10 , some respondents stated that this question was hard to comprehend. Although the data demonstrated a clear trend, it could still have errors in it. For the future, it would improve the accuracy of the results if the survey were tested with more people who did not participate in the final process. They can provide feedback and help enhance the survey.

\section{Conclusion}

In conclusion, the survey and analysis demonstrate the different changes influenced by COVID-19. The participants demonstrated that, due to COVID-19, they experience negative emotions more frequently and intensely. Their actions also 
display a decrease in consumption of goods and grocery store shopping as they opt for more online shopping. Americans do have a tendency of saving toilet paper due to their lack of supply and fears that they will not have enough. This phenomenon is especially intensified due to media reports as they publicize its great demand but lack of supply. On the other hand, another presumed essential, rice, was not over-purchased since there were no media outlets publicizing the purchases, leading people to have no fear of running out and missing out. Lastly, the respondents demonstrate that the three most important factors determining their purchases are now more realistic and necessity-driven than personal needs and satisfaction fulfillment.

This research project provides deeper insight into how a global health crisis can change the way people feel, think, and buy. Although future research is needed to perfect and correct the problems discovered during the process of this project, it provided a base for the questions regarding the emotional and behavioral influence of a pandemic.

\section{Methods}

The data summarized in this paper were collected through an online survey designed in two languages - English and Chinese. Using bilingual versions and websites, the survey receives responses from people who currently reside in China, the United States, Canada, and Australia (62.6\% live in China, 29.9\% live in the United States, $5.9 \%$ reside in Canada, and $1.6 \%$ reside in Australia). The motivation to create the survey in two languages is to target a wider group of respondents living in different countries speaking different languages. Both the English and Chinese versions have the same questions, with only one exception. The English version included a question asking whether the participants increased their stock of toilet paper. In contrast, the Chinese version questioned whether the participants increased their purchases of bags of rice. These two questions target the two daily-use items that people in different countries view as necessities. The survey was started on July 12th, 2020, and concluded two weeks later, on July 26th, 2020.

\section{Acknowledgement}

I would like to thank my parents for their endless support during this process. I am also grateful for Professor Yochanan Shachmurove's guidance throughout this project. Additionally, I am thankful for the team at IJHSR and all survey respondents. Without your help, I would not have completed this research.

\section{References}

1. COVID-19 Map, 2020. Johns Hopkins Coronavirus Resource Center. https://coronavirus.jhu.edu/map.html.

2. Coronavirus in the U.S.: Latest Map and Case Count, 2020. The New York Times. https://www.nytimes.com/ interactive/2020/us/coronavirus-us-cases.html.

3. New Cases of COVID-19 In World Countries, 2020.

John Hopkins Coronavirus Resource Center.

https://coronavirus.jhu.edu/data/new-cases.

4. Mental Health and Coping During COVID-19, 2020.

Centers for Disease Control and Prevention.

https://www.cdc.gov/coronavirus/2019-ncov/daily-life-

coping/managing-stress-anxiety.html.

5. ILO Monitor: COVID-19 and the World of Work. 4th Edition, 2020. International Labour Organization. https://www.ilo.org/global/topics/coronavirus/ impacts-and-responses/WCMS_745963/lang--en/ index.htm.

6. Soucheray, Stephanie. US Job Losses Due to COVID-19 Highest since Great Depression, 2020.

CIDRAP.https://www.cidrap.umn.edu/ news-perspective/2020/05/us-job-losses-duecovid-19-highest-great-depression.

7. Survey Finds Large Increase in Psychological Distress Reported among US Adults during the COVID-19 Pandemic, 2020. ScienceDaily. https://www.sciencedaily.com/ releases/2020/06/200603132550.htm

8. Restubog, Simon Lloyd D., Anna Carmella G. Ocampo, and Lu Wang. Taking Control amidst the Chaos:

Emotion Regulation during the COVID-19

Pandemic 2020. Journal of vocational behavior, 2020.

https://www.ncbi.nlm.nih.gov/pmc/articles/PMC7206430/

9. McGinty, Emma E., Rachel Presskreischer, Hahrie Han, and Colleen L. Barry. Psychological Distress and Loneliness Reported by US Adults in 2018 and April 2020, 2020. JAMA. https://jamanetwork.com/ journals/jama/fullarticle/2766941.

10. Jin, Xiaotong, Wei Song, Taiyang Zhao, and Feng Yao. The Impact of Public Health Event on Irrational Consumption Behavior of Residents, 2020 维普资讯 Journal of Xi'an Jiaotong University (Social Science). http://lib.cqvip.com/Qikan/Article/Detail?id=7102492717

11. Lee, Shawna J., and Kaitlin P. Ward. STRESS AND PARENTING DURING THE CORONAVIRUS PANDEMIC, 2020.

Parenting In Context Research Lab, 2020.

https://www.parentingincontext.

org/uploads/8/1/3/1/81318622/research_brief_stress_ and_parenting_during_the_coronavirus_pandemic_ final.pdf

12. Kluger, Jeffrey. Why Shoppers Are Hoarding Toilet Paper, 2020. TIME. https://time.com/5803273/hoardingtoilet-paper/.

13.Why the Pandemic Unleashed a Frenzy of Toilet-Paper Buying, 2020. Nature. https://www.nature.com/articles/ d41586-020-01836-1.

14. Carroll, Linda. Who Bought All the Toilet Paper? Study Suggests Who Was Most Likely to Stockpile during COVID-19, 2020.NBC. https://www.nbcnews. com/health/health-news/who-bought-all-toilet-paperstudy-suggests-who-was-most-n1230586.

15. Mittone, L., \& Savadori, L. The Scarcity Bias. Applied Psychology, 2009. 58(3), 453-468

16. Lofton, Justine. Why Are People Hoarding Toilet Paper during Coronavirus Pandemic? It's FOMO, 2020. MLIVE. https://www.mlive.com/coronavirus/2020/ 03/why-are-people-hoarding-toilet-paper-duringcoronavirus-pandemic-its-fomo.html

17. Mao, Frances. Coronavirus Panic: Why Are People Stockpiling Toilet Paper?, 2020. BBC. https://www.bbc.com/news/world-australia-51731422.

18. Wu, Zunyou, Quanyi Wang, Jing Zhao, Chun Huang, Zijian Feng, Jennifer M. McGoogan, and Peng Yang. Time Course of a Second Outbreak of COVID-19 in Beijing, China, June-July 2020, 2020. JAMA. https://jamanetwork.com/journals/jama 
fullarticle/2769930.

19. Scarcity, 2020. Wikipedia. https://en.wikipedia.org/ wiki/Scarcity.

20. Rabin, Roni Caryn. Many in China Wear Them, but Do Masks Block Coronavirus?, 2020. The New York Times. https://www.nytimes.com/2020/01/23/health/ coronavirus-surgical-masks.html.

21. Talev, Margaret. Ipsos Poll: The Coronavirus Death Toll Skeptics Are Growing, 2020. Axios. https://www.axios.com/axios-ipsos-poll-gop-skepticsgrowing-deaths-e6ad6be5-c78f-43bb-9230-

c39a20c8beb5.html?utm_source=twitter

22. Allyn, Bobby, and Barbara Sprunt. Poll: As

Coronavirus Spreads, Fewer Americans See

Pandemic As A Real Threat, 2020. NPR.

https://www.npr.org/2020/03/17/

816501871/poll-as-coronavirus-spreads-fewer-

americans-see-pandemic-as-a-real-threat.

\section{- Author}

Melissa Han is a senior at Flint Hill Upper School. She enjoys learning and studying social issues in the works of business. Melissa plans to double major in business and behavioral economics in college. 